\title{
Análisis Psicométrico Preliminar de la Escala de Actitudes Percibidas en Terapia hacia Personas Lesbianas, Gays y Bisexuales ${ }^{9}$
}

\author{
Caleb Esteban \\ PhD en Psicología Clínica \\ Ponce Health Sciences University, Puerto Rico \\ Correo electrónico: cesteban@psm.edu \\ Juan A. González Rivera \\ PsyD en Psicología Clínica \\ Ponce Health Sciences University, Puerto Rico \\ Correo electrónico: jagonzalez@psm.edu
}

\section{Margarita Francia Martínez \\ PhD en Psicología Clínica Universidad Carlos Albizu, Puerto Rico Correo electrónico: mfrancia@albizu.edu}

Recibido: $14 / 01 / 2020$

Evaluado: 31/01/2020

Aceptado: 13/02/2020

\section{Resumen}

En Puerto Rico, al igual que otros países, se viven actitudes negativas hacia las minorías sexuales y de género, especialmente hacia personas lesbianas, gay y bisexuales (LGB). Diversos estudios en la isla han encontrado actitudes negativas y distancia social de parte de profesionales de la psicología hacia la comunidad LGB. Sin embargo, no se tenía información sobre cómo las personas LGB percibían las actitudes en el espacio de terapia. Al no existir una escala para medir este fenómeno, se construye la Escala de Actitudes Percibidas en Terapia hacia Personas Lesbianas, Gays y Bisexuales. La muestra fue no probabilística y constituida por 104 participantes que se identificaban como LGB. Al revisar la distribución de los ítems de acuerdo a su carga factorial se identificaron dos factores: "Actitud Negativa" y "Actitud Positiva". Tanto la escala total, como los dos factores, mostraron coeficientes aceptables. En síntesis, los hallazgos preliminares de este estudio demostraron que la escala tiene la capacidad de examinar las actitudes de los terapeutas.

Palabras clave LGB/LGBT, psicoterapia, percepción, actitudes, validación.

9 Para citar este artículo: Esteban, C., González-Rivera, J. A. y Francia-Martínez, M. (2021). Análisis Psicométrico Preliminar de la Escala de Actitudes Percibidas en Terapia hacia Personas Lesbianas, Gays y Bisexuales. Informes Psicológicos, 21(2), pp. 145-160 http://dx.doi.org/10.18566/infpsic.v21n2a09 


\section{Preliminary Psychometric Analysis of the Scale of Perceived Attitudes in Therapy towards Lesbian, Gay and Bisexual People}

Abstract

In Puerto Rico, like other countries, there are negative attitudes towards sexual and gender minorities, especially towards lesbian, gay and bisexual (LGB) people. Various studies on the island have found negative attitudes and social distance on the part of psychology professionals towards the LGB community. However, there was no information on how LGB people perceived attitudes in the therapy space. As there is no scale to measure this phenomenon, the Scale of Perceived Attitudes in Therapy towards Lesbian, Gay and Bisexual People is constructed. The sample was non-probabilistic and made up of 104 participants who identified themselves as LGB. When reviewing the distribution of the items according to their factor load, two factors were identified: "Negative Attitude" and "Positive Attitude". Both the total scale and the two factors showed acceptable coefficients. In summary, the preliminary findings of this study demonstrated that the scale has the ability to examine the attitudes of therapists.

Keywords

LGB / LGBT, psychotherapy, perception, attitudes, validation.

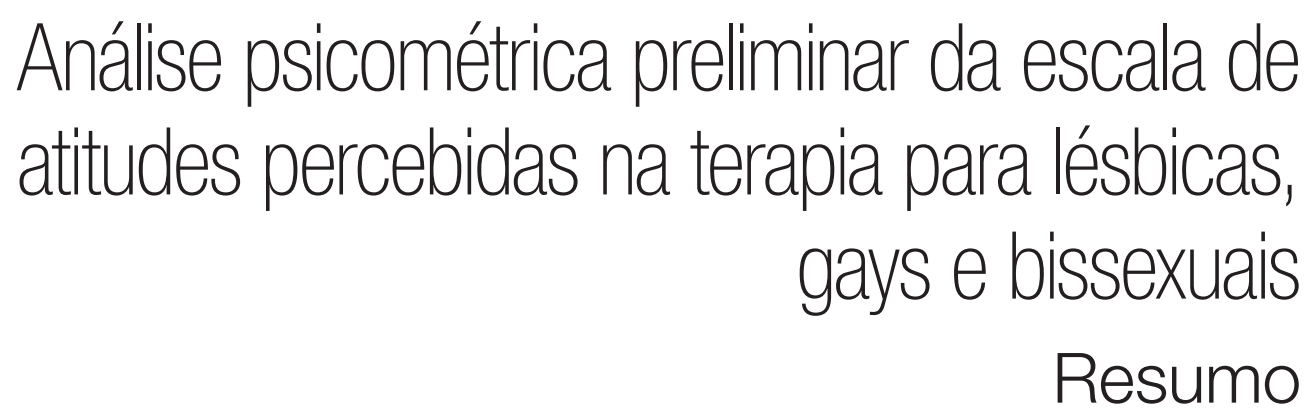

Em Porto Rico, como em outros países, existem atitudes negativas em relação às minorias sexuais e de gênero, especialmente em relação às pessoas lésbicas, gays e bissexuais (LGB). Vários estudos na ilha encontraram atitudes negativas e distanciamento social dos profissionais de psicologia em relação à comunidade LGB. No entanto, não havia informações sobre como as pessoas LGB percebiam as atitudes no espaço da terapia. Como não há escala para mensurar esse fenômeno, é construída a Escala de Atitudes Percebidas na Terapia para Lésbicas, Gays e Bissexuais. A amostra foi não probabilística e composta por 104 participantes que se identificaram como LGB. Ao revisar a distribuição dos itens de acordo com sua carga fatorial, foram identificados dois fatores: "Atitude Negativa" e "Atitude Positiva". Tanto a escala total quanto os dois fatores apresentaram coeficientes aceitáveis. Em resumo, os resultados preliminares deste estudo demonstraram que a escala tem a capacidade de examinar as atitudes dos terapeutas.

\section{Palavras chave}

LGB / LGBT, psicoterapia, percepção, atitudes, validação. 


\section{ntroducción}

En Puerto Rico, al igual que otros países del continente americano, se viven actitudes negativas hacia las minorías sexuales y de género, especialmente hacia la comunidad de personas lesbianas, gays, bisexuales e identidades trans (LGBT+). Un estudio hecho en la Isla midió las actitudes negativas hacia las personas lesbianas y gays en personas adultas puertorriqueñas que fueran padres o madres de familia. Este estudio encontró que la mayoría de las personas presentaban actitudes moderadas de prejuicio y alguna distancia social hacia las personas lesbianas y gays (Tirado-Martínez, 2018). Por otro lado, algunos estudios sobre prejuicios y actitudes negativas hacia miembros de la comunidad LGBT+ han hallado que estos prejuicios parecen disminuir a mayor educación en la vida (García, 1984; Toro-Alfonso \& Varas-Díaz, 2004), sin embargo, continúan siendo observados en estudiantes y profesionales de diversas disciplinas.

Las actitudes son respuestas naturales de los seres humanos y su función es brindar valores positivos, negativos o mixtos a un fenómeno (Abello-Forero et al., 2018). Las actitudes son un reflejo de nuestras creencias que incluye un componente cognitivo, conductual y afectivo hacia algo o alguien (Lilienfeld, Lynn, Namy, \& Woolf, 2011; Stangor, 2012), y son usualmente adquiridas por aprendizaje básico (AbelloForero et al., 2018). Las actitudes provienen y se mantienen por esquemas propios o replicas sociales y nos brindan información acerca de las características de una persona o un grupo (Stangor, 2012).
Las actitudes también han sido definidas como "un estado de la disposición nerviosa y mental, puesto que da respuesta a partir de las vivencias" (Bustos-Velasco, \& Rodríguez-Pinzón, 2016, p. 39). Varios estudios en Puerto Rico han indagado en las actitudes que se tienen hacia las personas gays y lesbianas principalmente. Por ejemplo, Toro-Alfonso y Varas-Díaz (2004) descubrieron niveles moderados de actitudes de prejuicio al encuestar estudiantes universitarios de la Universidad de Puerto Rico en San Juan. Casi una década más tarde, Nieves-Rosa (2012) halló, en estudiantes universitarios de la Universidad de Puerto Rico en Mayagüez, altos niveles de actitudes de prejuicio y una distancia social moderada hacia personas lesbianas y gays. Más adelante, Fernández-Rodríguez y Calderón-Squiabro (2014) igualmente descubrieron actitudes moderadas de prejuicio y distancia social hacia las personas gay y lesbianas en la Universidad de Puerto Rico en Cayey.

Por otro lado, a nivel profesional Barbosa-Hernández (2013) identificó actitudes moderadas de prejuicio hacia personas lesbianas y gays en profesionales de la enseñanza superior del país. Al evaluar las competencias autopercibidas por profesionales de la consejería en rehabilitación para trabajar con personas gays, lesbianas y bisexuales, Rivera-Colón, Ramos-Reyes y Vázquez-Ramos (2019) notaron que las personas participantes mostraron competencias moderadas en las sub-dimensiones del instrumento en conocimiento, destrezas y actitudes.

En cuanto a la disciplina de la psicología, Vázquez-Rivera, Nazario-Serrano y Sayers-Montalvo (2012) exploraron las actitudes de estudiantes posgrado de psicología clínica y profesionales de la 
psicología clínica con licencia. En este estudio se reportó que solo el $5 \%$ y $6 \%$ de la muestra prefieren no atender a clientes gay y lesbianas, mientras que el $13 \%$ y $6 \%$ de estudiantes y profesionales respectivamente indicaron no ser competentes para atender a esta población. Por la misma vía, Vázquez-Rivera, Esteban y Toro-Alfonso (2018) en un estudio solo con profesionales de la psicología con licencia en la isla examinaron más a detalle las actitudes negativas hacia personas gay y lesbianas. Detectaron que la mayoría de las personas participantes reportaron actitudes positivas hacia la comunidad, poca distancia social y niveles bajos de prejuicio. Sin embargo, dieron con una minoría de estos profesionales con altos niveles de prejuicio. Datos similares se han hallado cuando se exploran las actitudes y distancia social hacia la comunidad trans. Un estudio llevado a cabo por Francia-Martínez, Esteban y Lespier (2016) con estudiantes posgraduados de psicología y profesionales de la psicología en Puerto Rico, reflejaron que un $14.9 \%$ de la muestra mostró prejuicios moderados hacia la comunidad transgénero y un $19.8 \%$ hacia la comunidad transexual. En cuanto a distancia social, un $6.4 \%$ reportó distancia moderada y un $43.2 \%$ distancia moderada baja.

Por otro lado, el conocer una persona o un grupo influencia las creencias, tanto a nivel cognitivo, conductual y afectivo, hacia esa persona o grupo. Estudios que han explorado las actitudes y conocimiento hacia las personas homosexuales y bisexuales han expuesto que existe una relación positiva entre mayor conocimiento y actitudes positivas (Franco-Morales et al., 2016). Además, Esteban et al. (2019) encuestaron una muestra de personas LGB que habían asistido a terapia, y encontraron dos correlaciones significativas: mientras más conocimiento sobre la comunidad LGB de parte de la persona terapeuta, y mayores actitudes positivas percibidas por parte del terapeuta, mayor era la satisfacción en el proceso de terapia.

Los instrumentos para medir destrezas en terapia han sido amplios y en una diversidad de constructos (Maurino et al., 2018). No obstante, han sido escasos los instrumentos en las últimas décadas con el propósito de medir las competencias, actitudes y conocimientos de terapeutas hacia la comunidad LGB. Dillon y Worthington (2003) crearon un inventario para medir la autoeficacia al trabajar la consejería afirmativa con Lesbianas, Gays y Bisexuales (Lesbian, Gay, and Bisexual Affirmative Counseling Self-Efficacy Inventory, LGBCSI). Este inventario medía las competencias de autoeficacia en las dimensiones de la aplicación de conocimientos, las habilidades de abogacía, la autoconsciencia, las destrezas en relaciones y las destrezas de evaluación. Luego de llevar a cabo análisis con cinco diferentes estudios, documentaron una consistencia interna alta, pero una fiabilidad bajo de test-retest (dos semanas después). Luego, Dillon et al. (2015) publican la versión corta de este inventario (Lesbian, Gay, and Bisexual Affirmative Counseling Self-Efficacy Inventory - Short Form, LGB-CSI-SF) consistiendo en 3 reactivos de sus 5 componentes. En esta ocasión, el inventario continuó con una consistencia interna alta, pero con una fiabilidad adecuada de test-retest (una semana después).

Bidell (2005) creó la Escala de Competencias de Consejeros sobre Orientación Sexual (Sexual Orientation Counselor Competency Scale, SOCCS) midiendo las competencias autopercibidas en las dimensiones de actitudes, destrezas, conocimiento 
al trabajar consejería con clientes lesbianas, gays y bisexuales. Con una muestra de estudiantes y profesionales de la consejería, esta escala obtuvo un coeficiente de alpha para su escala total de $.90, .88$ para la dimensión de actitudes, .91 para la dimensión de destrezas y .76 para la dimensión de conocimiento. Además, contó con un análisis test-retest de fiabilidad (una semana después) para un coeficiente de .84 para la escala general. Luego de unos años, Graham, Carney y Kluck (2012) utilizaron esta misma escala con estudiantes a nivel graduado de consejería educativa y consejería psicológica. En esta ocasión, la SOCCS obtuvo un coeficiente de alpha para su escala total de .87, .91 para la dimensión de actitudes, .86 para la dimensión de destrezas y .71 para la dimensión de conocimiento.

Worthington, Dillon y Becker-Schutte (2005) crearon una escala para medir el conocimiento y las actitudes de personas heterosexuales hacia las personas lesbianas, gays y bisexuales (Lesbian, Gay, and Bisexual Knowledge and Attitudes Scale for Heterosexuals, LGB-KASH). Esta escala mide afirmatividad internalizada, actitudes hacia los derechos civiles, conocimiento, conflictos religiosos y odio. Luego de cuatro estudios, los análisis de validez sustentan el uso de esta escala, pero se recomienda continuar trabajando en su construcción.

Finalmente, tanto Vázquez-Rivera et al. (2018) como Francia-Martínez et al. (2016) concluyen en sus discusiones que, en estos estudios por disponibilidad, pareciera que solo aquellas personas profesionales y estudiantes con actitudes positivas son las que participan. Por lo tanto, la mejor manera para corroborar las actitudes que tienen las personas terapeutas en terapia es preguntándole a la misma comunidad. Sin embargo, luego de una revisión de literatura, se encontraron instrumentos que miden actitudes, pero estaban creados para que fuera el mismo profesional quien evalúe sus percepciones sobre sus competencias, eficacia, actitudes y/o conocimientos. Por esta razón, se hizo imprescindible la creación de un instrumento que midiera el otro lado del fenómeno, el explorar las actitudes percibidas por la misma comunidad LGB hacia sus terapeutas en el proceso de terapia.

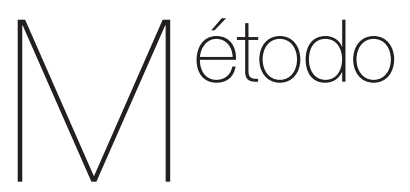

\section{Diseño de Investigación}

Esta investigación tuvo un diseño no experimental de tipo instrumental (Ato, López, \& Benavente, 2013; Montero \& León, 2007). Este diseño se utiliza en aquellas investigaciones que desean examinar las propiedades psicométricas de pruebas o medidas psicológicas. En esta investigación se desarrolló y validó preliminarmente la Escala de Actitudes Percibidas en Terapia hacia Lesbianas, Gays y Bisexuales en una muestra de personas que autoidentificaban su orientación sexual como lesbiana, gay, o bisexual.

\section{Participantes}

Los análisis psicométricos se realizaron con una muestra no probabilística compuesta por 104 personas que se identificaban como lesbiana, gay o bisexual. 
Estas personas participaron confidencial y voluntariamente en la investigación. La edad promedio de la muestra fue 29.18 $(\mathrm{DE}=8.47)$. La Tabla 1 presenta la descripción sociodemográfica de las personas participantes, quienes debían cumplir con los siguientes criterios de inclusión: (a) mayores de 21 años de edad, (b) residir en Puerto Rico (c) haber recibido servicios de psicoterapia e, (d) identificar su orientación sexual como homosexual o bisexual. Tres personas participantes se definieron como pansexuales, las cuales fueron incluidas bajo la sombrilla bisexual.

Tabla 1.

Características Sociodemográficos de los Participantes

\begin{tabular}{|c|c|c|}
\hline Variable & $f$ & $\%$ \\
\hline \multicolumn{3}{|l|}{ Sexo } \\
\hline Hombre & 56 & 53.8 \\
\hline Mujer & 48 & 46.2 \\
\hline \multicolumn{3}{|l|}{ Género } \\
\hline Masculino & 55 & 52.9 \\
\hline Femenino & 43 & 41.3 \\
\hline Género no-conforme & 4 & 3.8 \\
\hline Bigénero & 2 & 1.9 \\
\hline \multicolumn{3}{|l|}{ Orientación sexual } \\
\hline Gay & 48 & 46.2 \\
\hline Lesbiana & 28 & 27.0 \\
\hline Bisexual & 25 & 24.0 \\
\hline Pansexual & 3 & 2.8 \\
\hline \multicolumn{3}{|l|}{ Estatus Marital } \\
\hline Soltería & 52 & 50.0 \\
\hline Con pareja no conviviendo & 25 & 24.0 \\
\hline Con pareja conviviendo & 20 & 19.2 \\
\hline Matrimonio & 4 & 3.9 \\
\hline En separación & 1 & 1.0 \\
\hline Relación poliamorosa & 1 & 1.0 \\
\hline Missing & 1 & 1.0 \\
\hline
\end{tabular}

\begin{tabular}{lll}
\hline Variable & $\mathbf{f}$ & $\%$ \\
\hline Afiliación religiosa & & \\
\hline Ninguna & 54 & 51.9 \\
Católica & 21 & 20.2 \\
Cristiana & 13 & 12.5 \\
Protestante/Evangélica & 10 & 9.6 \\
Espiritismo/Santería & 4 & 3.9 \\
Musulmana & 1 & 1.0 \\
Missing & 1 & 1.0 \\
\hline
\end{tabular}

\begin{tabular}{lcc} 
Ingreso económico anual (dólares) & & \\
\hline Ninguno & 20 & 19.2 \\
Menos de $\$ 12,000$ & 40 & 38.5 \\
$\$ 12,001$ a $\$ 32,000$ & 24 & 23.1 \\
$\$ 32,001$ a $\$ 52,000$ & 11 & 10.6 \\
$\$ 52,001$ a $\$ 72,000$ & 6 & 5.8 \\
$\$ 72,001$ a $\$ 92,000$ & 1 & 1.0 \\
Mayor a $\$ 92,001$ & 2 & 1.9 \\
\hline Educación & & \\
\hline Escuela secundaria & 10 & 9.6 \\
Cursos cortos/técnicos & 3 & 2.9 \\
Grado asociado & 3 & 2.9 \\
Grado de Bachiller & 50 & 48.1 \\
Grado de Maestría & 27 & 26.0 \\
Grado Doctoral & 11 & 10.6 \\
\hline
\end{tabular}

Nota. $n=104$

\section{Instrumentos}

Planilla de datos generales. Las personas participantes completaron una planilla que recolectaba alguna información sociodemográfica. Específicamente, preguntaba sobre el sexo, edad, género, orientación sexual, estatus marital, afiliación religiosa, ingreso económico anual y grado académico de las personas participantes. 


\section{Escala de Actitudes Percibidas en Terapia hacia Lesbianas, Gays y Bisexuales.}

Esta escala es un instrumento generado por el equipo de investigación. En un comienzo ésta tuvo 36 reactivos con dos subescalas: actitudes positivas y actitudes negativas hacia la comunidad LGB en el espacio de la terapia. Para la validez de contenido, 12 jueces y juezas (jurado) con experiencia en investigación y con conocimiento de la comunidad LGBT evaluaron los reactivos, con el objetivo de identificar si estos eran pertinentes a las actitudes hacia personas LGB en un contexto terapéutico (Método Lawshe). Para rechazar o retener los ítems se usó el Content Validity Ratio (CVR). Para interpretar los resultados, utilizamos los valores críticos recalculados por Wilson et al. (2012). De acuerdo con estas personas autoras, el valor mínimo requerido para las 12 personas del jurado según la tabla de Schipper es .57 para aceptar un ítem como esencial. Solo se eliminaron dos elementos por estar bajo la adecuación de CVR. Las respuestas a los ítems se realizaron en una escala Likert de 5 puntos $(1=$ totalmente en desacuerdo hasta 5 = totalmente de acuerdo).

\section{Procedimientos Generales}

El presente estudio fue evaluado y aprobado por el Institutional Review Board de la Universidad Carlos Albizu de San Juan, Puerto Rico (Sum-16-05). Luego se procedió a recopilar la información de forma electrónica, a través de la página web de SurveyMonkey. Al acceder dicha página, las personas participantes leían el consentimiento informado, donde no era necesario identificarse con su nombre, que explicaba a las personas interesadas en participar: el objetivo del estudio, los riesgos, beneficios, el tiempo de la participación y su derecho a abandonar el estudio en el momento que desee (para lo cual solo debía cerrar su buscador de internet). Para correr los análisis estadísticos, se utilizó el paquete IBM SPSS (IBM Corporation, 2016). Como parte de los análisis, se realizaron pruebas descriptivas, análisis factoriales, índices de discriminación de ítems y se calcularon coeficientes de confiabilidad.

El análisis factorial exploratorio (AFE) se realizó utilizando el método de extracción de ejes principales con rotación oblicua para identificar las variables latentes que miden el conjunto de ítems. Este procedimiento no requiere que los datos se distribuyan de forma normal (Fabrigar, Wegener, MacCallum, \& Strahan, 1999) y, en estos casos, es más exacto que la rotación ortogonal (Schmitt, 2011). Para identificar la cantidad de factores se emplearon dos técnicas: el Scree Test (prueba de sedimentación) y la varianza explicada por los factores extraídos en el AFE. Hatcher (1994) recomienda que únicamente se reconozcan como adecuados los factores que expliquen como mínimo un 5\% de la varianza. Al realizar el AFE, solo dos factores cumplieron adecuadamente este supuesto. Por su parte, Stevens (2002) sugiere que se reconozcan como aceptables ítems con cargas factoriales superiores a .50 .

Los índices de discriminación de los ítems se calcularon a través de la correlación entre los ítems y la escala total 
$\left(r_{\text {bis }}\right)$. Según Field (2013), estos índices deben ser mayores a .30 para ser reconocidos como aceptables. Además, la confiabilidad del instrumento se examinó a través del coeficiente Alfa de Cronbach. DeVellis (2017), uno de los investigadores más reconocidos en temas relacionados al desarrollo de instrumentos de medición, indica que valores alfa superiores a .70 son aceptables, valores entre .80 y .90 son buenos, y valores superiores a .90 son indicativos de que la escala debe revisarse o reducirse. Asimismo, Campo-Arias y Oviedo (2008) indican que valores mayores a .90 son indicativos de duplicidad de ítems o redundancia en las ideas de los mismos. En estos casos, se recomienda revisar los ítems y eliminar los ítems que repiten ideas.

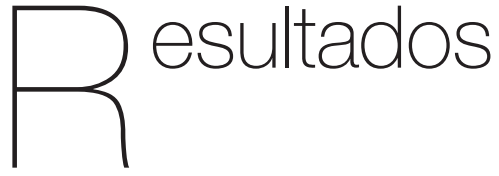

El primer AFE evidenció que solo dos factores explicaban más del $5 \%$ de la varianza, $K M O=.897 ; X^{2}(630)=3097.676, p$ $<$.001. Asimismo, los resultados del Scree Test apoyan la retención de dos factores (véase Figura 1). Por esta razón, realizamos un segundo AFE con restricción a dos dimensiones, donde de los 36 ítems se descartaron cinco por no alcanzar los criterios de inclusión (2, 3, 18, 23, 34). Los 31 ítems restantes se dividieron de la siguiente forma: 23 ítems en el factor 1 y 8 ítems en el factor 2. La Tabla 2 muestra las cargas factoriales de ambos factores.

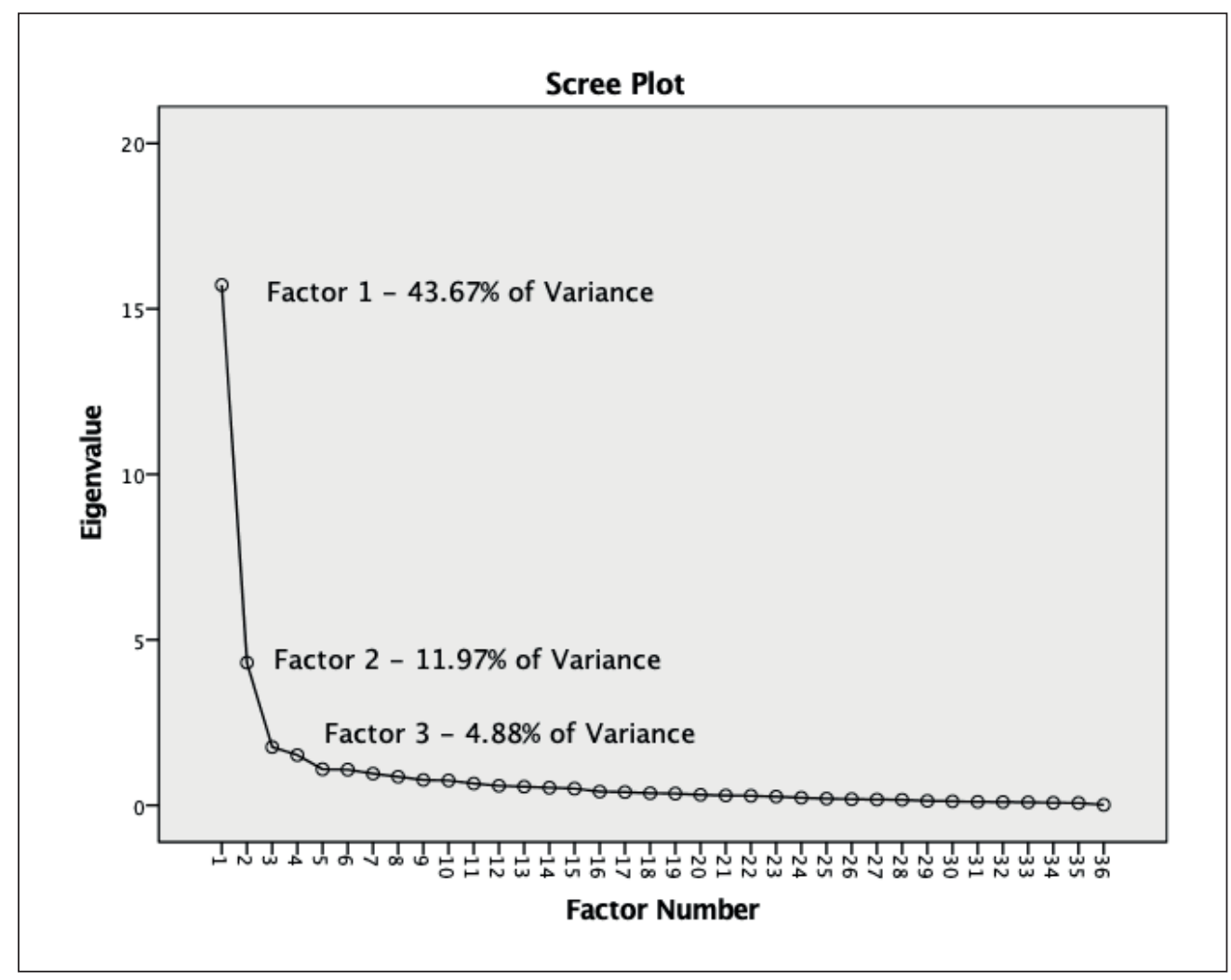

Figura 1. Gráfica de sedimentación y varianza explicada por los primeros factores 
Tabla 2

Distribución Factorial del Segundo AFE

1. Mi terapeuta se mostró incómodo cuando me preguntaba sobre mi orientación sexual.

1

4. Mi terapeuta mostró comodidad al hablar sobre mis diversas experiencias sexuales.

5. Mi terapeuta mostró conocimiento sobre la cultura LGB.

6. Mi terapeuta exploró mi orientación sexual desde el inicio de la terapia.

7. Mi terapeuta evitó el tema de sexualidad sabiendo que era homosexual o bisexual.

8. Mi terapeuta utilizó terminología adecuada para referirse a mi orientación sexual.

9. Mi terapeuta apoyó los procesos de divulgación de mi orientación sexual.

10. Mi terapeuta me explicó que las actitudes negativas de la sociedad por orientación...

11. Mi terapeuta exploró cómo las actitudes negativas de la sociedad han tenido...

12. Mi terapeuta reconoció las situaciones específicas de las personas LGB.

13. Mi terapeuta reaccionó negativamente cuando le dije que me atraían las personas...

14. Mi terapeuta realizó comentarios negativos cuando le dije que me atraían las...

15. Mi terapeuta integró el tema de mi orientación sexual en la terapia.

16. Mi terapeuta me cuestionó si estaba seguro de mi orientación sexual.

17. Mi terapeuta sugirió que tuviese (o continuara) relaciones heterosexuales para...

19. Mi terapeuta exploró sobre mis experiencias sexuales del pasado.

20. Mi terapeuta validó mis experiencias sexuales como algo normal.

21. Mi terapeuta tenía clara la diferencia entre orientación sexual e identidad de género.

22. Mi terapeuta utilizaba estereotipos relacionados a mi orientación sexual.

24. Mi terapeuta mostraba conocimiento de las implicaciones de tener una pareja del...

25. Mi terapeuta entendía el impacto que podría tener mi orientación sexual en mi...

26. Mi terapeuta tomó en consideración la armonía entre mis creencias espirituales y...

27. Mi terapeuta exploró sobre enfermedades de transmisión sexual, como lo haría...

28. Mi terapeuta estuvo consciente del impacto de ser LGB en otros ámbitos de mi...

29. Mi terapeuta me informó sobre su experiencia, educación y/o adiestramiento sobre...

30. Mi terapeuta me informó sobre recursos de apoyo apropiados para la comunidad LGB.

31. Mi terapeuta demostró estar al día sobre los movimientos de derechos LGB.

32. Los documentos de la oficina tenían lenguaje inclusivo para la comunidad LGB.

33. Mi terapeuta se mostró sensible a las situaciones asociados a mi orientación sexual.

35. Sentía confianza en llevar a la terapia asuntos relacionados a la sexualidad.

.77

.59

.53

.52

.52

.59

.77

.78

.82

.91

.88

.66 
Los ítems de cada factor se examinaron mediante la correlación ítem-total (rbis) y el alfa de Cronbach. El primer factor, dado que quedo configurado por 23 ítems, fue examinado con criterios más estrictos de selección. Siguiendo las recomendaciones de Field (2013) para estos casos, se mantuvieron aquellos ítems cuyos valores fuesen mayores a $.30 \mathrm{y}$ menores a .70. Los resultados apoyaron la eliminación de once ítems $(4,5,9,12$, 21, 24, 25, 28, 31, 33 y 35). Esto redujo el factor 1 a 12 ítems. Por su parte, los ocho ítems pertenecientes al factor 2 mostraron índices de discriminación superiores a .30. Sin embargo, dado que el factor 2 presentó un alfa de Cronbach superior a .90 ( $\alpha=.92)$, se examinaron los ítems para determinar duplicación de ideas en los mismos. Los ítems 13 y 14 miden aspectos semejantes entre sí, por lo cual se eliminó el ítem 14. Esto disminuyó el factor 2 a siete ítems. Estos 19 ítems fueron sometidos nuevamente a un AFE y al cálculo del índice de correlación ítem-total.
Este nuevo AFE evidenció una estructura bifactorial que explicaba el 53.59\% de la varianza. No obstante, siete ítems no cumplieron con los criterios de selección, por lo cual se eliminaron (ítems 6, 7, 8, 15, 26, 32, 20). Un último análisis factorial exploratorio mostró nuevamente una estructura bifactorial que explicaba el $57.54 \%$ de la varianza, donde el $35.13 \%$ era explicado por el factor 1 y el $22.41 \%$ por el factor 2 , $K M O=.827 ; X^{2}(66)=659.977, p<.001$. Esta vez todos los ítems cumplieron con los criterios de inclusión. El análisis de discriminación reveló que todos los ítems presentaron índices superiores a .30, tanto en su respectivo factor como en la escala total. En la Tabla 3 se presentan las cargas factoriales y los índices de discriminación obtenidos por los ítems en su respectivo factor: "Actitud Negativa" y "Actitud Positiva". Luego, se analizó la confiabilidad de la escala final y de sus dos factores mediante el alfa de Cronbach y el alfa de Cronbach estandarizado. Tanto la escala total como los dos factores, mostraron coeficientes aceptables (ver Tabla 4).

Tabla 3.

Cargas Factoriales e Índices de Discriminación de los Dos Factores

\begin{tabular}{|c|c|c|c|c|}
\hline Ítem & 1 & 2 & $r_{\text {bis } F}$ & $r_{\text {bis } T}$ \\
\hline $\begin{array}{l}\text { 13. Mi terapeuta reaccionó negativamente cuando le dije que me atraían las } \\
\text { personas de mi mismo sexo. }\end{array}$ & .84 & & .79 & .57 \\
\hline $\begin{array}{l}\text { 17. Mi terapeuta sugirió que tuviese (o continuara) relaciones heterosexuales } \\
\text { para estar seguro de mi orientación sexual. }\end{array}$ & .82 & & .76 & .49 \\
\hline 22. Mi terapeuta utilizaba estereotipos relacionados a mi orientación sexual. & .82 & & .78 & .52 \\
\hline $\begin{array}{l}\text { 1. Mi terapeuta se mostró incómodo cuando me preguntaba sobre mi } \\
\text { orientación sexual. }\end{array}$ & .81 & & .77 & .66 \\
\hline $\begin{array}{l}\text { 36. Me provocó ansiedad la forma en que mi terapeuta indagó sobre } \\
\text { asuntos de mi sexualidad. }\end{array}$ & .70 & & .69 & .59 \\
\hline 16. Mi terapeuta me cuestionó si estaba seguro de mi orientación sexual. & 69 & & .61 & .31 \\
\hline $\begin{array}{l}\text { 10. Mi terapeuta me explicó que las actitudes negativas de la sociedad por } \\
\text { orientación sexual pueden causarme malestar psicológico. }\end{array}$ & & .72 & .64 & .56 \\
\hline $\begin{array}{l}\text { 11. Mi terapeuta exploró cómo las actitudes negativas de la sociedad han } \\
\text { tenido impacto en mi vida. }\end{array}$ & & .71 & .64 & .55 \\
\hline
\end{tabular}




\begin{tabular}{|c|c|c|c|c|}
\hline Ítem & 1 & 2 & $r_{\text {bis } F}$ & $r_{\text {bis } T}$ \\
\hline $\begin{array}{l}\text { 29. Mi terapeuta me informó sobre su experiencia, educación y/o } \\
\text { adiestramiento sobre el tema de orientación sexual. }\end{array}$ & & .71 & .60 & .50 \\
\hline $\begin{array}{l}\text { 30. Mi terapeuta me informó sobre recursos de apoyo apropiados para la } \\
\text { comunidad LGB. }\end{array}$ & & .70 & 63 & .51 \\
\hline 19. Mi terapeuta exploró sobre mis experiencias sexuales del pasado. & & .65 & 62 & .33 \\
\hline $\begin{array}{l}\text { 27. Mi terapeuta exploró sobre enfermedades de transmisión sexual, como } \\
\text { lo haría con una persona heterosexual. }\end{array}$ & & .58 & .49 & .43 \\
\hline
\end{tabular}

Nota. Factor 1 = Actitud negativa; Factor 2 = Actitud positiva; $r_{b i s F}=$ índice de discriminación del ítem en su respectivo factor; $r_{b i s}=$ índice de discriminación del ítem para la escala total. $(n=104)$.

Tabla 4.

Medias, Desviaciones Estándar y Alfas

\begin{tabular}{ccccc}
\hline Factor & M & DE & $\alpha$ & $\alpha_{\text {est }}$ \\
\hline Actitud Negativa & 19.75 & 6.15 & .89 & .90 \\
\hline Actitud Positiva & 20.90 & 7.61 & .83 & .83 \\
\hline Escala Total & 45.72 & 8.99 & .83 & .84 \\
\hline
\end{tabular}

Nota. $\mathrm{M}=$ media; $\mathrm{DE}=$ desviación estándar; $\alpha$ = alfa de Cronbach; $\alpha_{\text {est }}=$ alfa de Cronbach estandarizado.

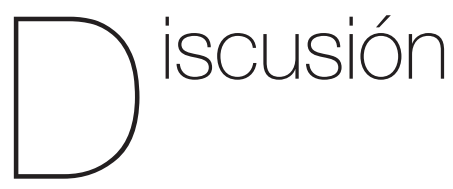

El presente estudio tuvo como propósito desarrollar y validar preliminarmente la Escala de Actitudes Percibidas en Terapia hacia Personas Lesbianas, Gays y Bisexuales. Este instrumento es el primero que evalúa las actitudes percibidas por la misma comunidad LGB hacia sus terapeutas en el proceso de terapia. La versión final quedó conformada por 12 ítems (ver Apéndice 1). Los resultados demostraron que el instrumento posee propiedades psicométricas adecuadas para evaluar este fenómeno desde un modelo de dos factores: Actitudes positivas y Actitudes negativas. Los factores examinan de forma fiable aspectos particulares de las actitudes percibidas por la comunidad LGB sobre sus terapeutas, sin que los factores redunden entre sí. Los índices de confiabilidad sugieren, como indica DeVellis (2017), que el instrumento muestra una sólida consistencia interna y es recomendable para utilizarse como instrumento de medición científica en investigaciones, tanto en Puerto Rico como en el Caribe. Además, la correlación entre los ítems y la escala total son indicadores plausibles de discriminación y cuentan con la capacidad de diferenciar personas con distintas actitudes en terapia.

En términos teóricos, los hallazgos psicométricos demostraron que el instrumento tiene la capacidad de examinar las actitudes de las personas terapeutas desde dos manifestaciones típicas: actitud negativa y actitud positiva. El factor actitud negativa incluye ítems que reflejan prejuicios y actitudes negativas hacia lesbianas, gays y bisexuales en terapia. Específicamente, este factor evalúa 
incomodidad del terapeuta (ítem 1), reacciones negativas (ítem 4), cuestionamiento inadecuado (ítem 5), recomendaciones heterosexuales (ítem 6), utilización de estereotipos (ítem 8) y provocación de ansiedad en la persona atendida (ítem 12). La literatura científica ha asociado consistentemente el prejuicio y las actitudes negativas hacia LGB con deterioros significativos en la salud mental (Burges, Lee, Tran, \& Van Ryn, 2007; Sandfort, de Graaf, \& Bijl, 2003; Singh \& Dickey, 2016; Woodford, Paceley, Kulick, \& Hung, 2015). Es decir, una persona terapeuta con actitudes negativas hacia sus pacientes, agrava la situación de salud mental del mismo. Por su parte, el factor de actitud positiva agrupó todos los ítems que reflejan respeto y competencias adecuadas para trabajar con personas lesbianas, gays y bisexuales en terapia. En la escala, este factor examina psicoeducación adecuada a su paciente (ítem 2), exploración adecuada de información (ítem 3), indagación de historial sexual (ítem 7 y 9), informar sobre experiencia y adiestramiento en orientación sexual (ítem 10) y recursos de apoyos disponibles (ítem 11).

En términos pragmáticos, la versión final de la Escala de Actitudes Percibidas en Terapia hacia Personas Lesbianas, Gays y Bisexuales (ver Apéndice 1) es adecuada para desarrollar nuevos estudios en la comunidad LGB caribeña e incluso latinoamericana. Este es un gran adelanto en la profesión dado que, en América Latina, no existía una escala que evaluara este fenómeno. El lenguaje accesible y lo breve de la escala permiten que sea de fácil administración, evaluación e interpretación. En cuanto su interpretación, recomendamos dos formas distintas: sumando los valores de las respuestas de los 12 ítems, para calcular un índice global de actitudes percibidas en terapia hacia LGB, o sumando los seis ítems de cada factor por separado. Los ítems pertenecientes al factor actitud negativa se puntúan de forma inversa. Los ítems se ordenaron de forma aleatoria en la versión final. Las puntuaciones posibles en la escala final fluctúan de 12 a 60; a mayor actitud positiva percibe la persona LGB sobre su terapeuta.

Este estudio cuenta con algunas limitaciones que valen la pena enumerar. En primer lugar, la muestra fue por disponibilidad, es decir, no contamos con un muestro aleatorio, debido a las particularidades de la población. En segundo lugar, no se examinó la confiabilidad de la escala a través del tiempo, solo mediante el análisis de su estructura interna. Por último, la recopilación de los datos no se realizó de forma estandarizada, lo que puede acrecentar el error estándar de la medida. Más allá de estas limitaciones, el estudio cuenta con dos bondades que deben ser resaltadas. En primer lugar, es la primera escala desarrollada y validada en el Caribe y América Latina para medir este fenómeno. En segundo lugar, aporta a la comunidad de investigadores de habla hispana una escala con excelentes propiedades psicométricas que ayudará a entender cómo los miembros de la comunidad LGB perciben el prejuicio por parte de sus terapeutas. Futuros estudios deben administrar la escala a otra muestra de participantes, aleatoria preferiblemente, para realizar el proceso de validación cruzada. Además, se debe analizar la confiabilidad temporal de la escala y realizar un análisis factorial confirmatorio. Por último, se recomienda que la Escala de Actitudes Percibidas en Terapia hacia Personas Lesbianas, Gays y Bisexuales se valide en otros países. 


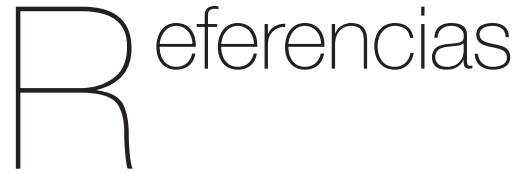

Abello-Forero, E., Bello-Pulido, J., GüizaSuarez, A., Ortiz-Escobar, A.M., RiverosMunévar, F., Vera-Maldonado, L.A., BernalVásquez, A.P., \& Reyes-Gómez, L. (2018). Actitudes hacia los niños adoptables por parte de familias monoparentales y nucleares de estratos 4, 5 y 6 en la ciudad de Bogotá. Informes Psicológicos, 18(2), 45-62. http://dx.doi.org/10.18566/infpsic. v18n2a03

Ato, M., López, J. J., \& Benavente, A. (2013). Un sistema de clasificación de los diseños de investigación en psicología. Anales de Psicología, 29(3), 1038-1059. http://dx.doi. org/10.6018/analesps.29.3.178511

Barbosa-Hernández, A. M. (2013). Actitudes de maestros de escuela intermedia y superior ante adolescentes gays y lesbianas, un estudio exploratorio. (Disertación doctoral no publicada). Universidad Carlos Albizu, San Juan, Puerto Rico.

Bidell, M. P. (2005). The Sexual Orientation Counselor Competency Scale: Assessing attitudes, skills, and knowledge of counselors working with lesbian, gay, and bisexual clients. Counselor Education and Supervision, 44(4), 267-279. https://doi. org/10.1002/j.1556-6978.2005.tb01755.x

Burges, D., Lee, R., Tran, A., \& Van Ryn, M. (2007). Effects of perceived discrimination on mental health and mental health services utilization among gay, lesbian, bisexual and transgender persons. Journal of LGBT Health Research, 3(4), 1-14. https://doi.org /10.1080/15574090802226626
Bustos-Velasco, V. A., \& Rodríguez-Pinzón, H. Y. (2016). Estructura dimensional de una escala de actitudes hacia la estadística en estudiantes universitarios de segundo y tercer semestre de la facultad de psicología de la Universidad Piloto de Colombia. Informes Psicológicos, 16(2), 37-52. http:// dx.doi.org/10.18566/infpsicv16n2a03

Campo-Arias, A., \& Oviedo, H. C. (2008). Propiedades psicométricas de una escala: La consistencia interna. Revista de Salud Pública, 10(5), 831-839. https://doi. org/10.1590/s0124-00642008000500015

DeVellis, R.F. (2017). Scale development: Theory and applications (4th Ed). California: Sage Publications.

Dillon, F. R., Alessi, E. J., Craig, S., Ebersole, R. C., Kumar, S. M., \& Spadola, C. (2015). Development of the Lesbian, Gay, and Bisexual Affirmative Counseling SelfEfficacy Inventory - short form (LGB-CSISF). Psychology of Sexual Orientation and Gender Diversity, 2(1), 86-95. https://doi. org/10.1037/sgd0000087

Dillon, F., \& Worthington, R. L. (2003). The Lesbian, Gay and Bisexual Affirmative Counseling Self-Efficacy Inventory (LGBCSI): Development, validation, and training implications. Journal of Counseling Psychology, 50(2), 235-251. https://doi. org/10.1037/0022-0167.50.2.235

Esteban, C., Francia-Martínez, M., VázquezRivera, M., Crespo, F., Bruno-Ortiz, T., \& Santiago-Ortiz, A. M. (2019). Are we doing OK?: Perception of satisfaction of $L G B$ clients in the therapy process. Manuscrito sometido para publicación.

Fabrigar, L. R., Wegener, D. T., MacCallum, R. C., \& Strahan, E. J. (1999). Evaluating 
the use of exploratory factor analysis in psychological research. Psychological Methods, 4(3), 272-299. http://dx.doi. org/10.1037/1082-989X.4.3.272

Fernández-Rodríguez, M., \& CalderónSquiabro, J. (2014). Prejuicio y distancia social hacia personas homosexuales por parte de jóvenes universitarios. Revista Puertorriqueña de Psicología, 25(1), 52-60.

Field, A. P. (2013). Discovering statistics using SPSS. London, England: SAGE.

Francia-Martínez, M., Esteban, C., \& Lespier, Z. (2016). Actitudes, conocimiento y distancia social de psicoterapeutas con la comunidad transgénero y transexual. Revista Puertorriqueña de Psicología, 28(1), 98-113.

Franco-Morales, F., Correa-Molina, E., Venet, M., \& Pérez-Bedoya, S. (2016). Relación actitudes-conocimientos sobre diversidad sexual en una muestra universitaria colombiana. Revista Internacional de Investigación en Educación, 8(17), 135-156. https://doi.org/10.11144/Javeriana.m8-17. rads

García, H. (1984). Actitudes hacia homosexuales y las lesbianas de cuatro grupos de estudiantes universitarios. (Tesis de Maestría) Universidad de Puerto Rico, Río Piedras, Puerto Rico.

Graham, S. R., Carney, J. S., \& Kluck, A. S. (2012). Perceived competency in working with LGB clients: Where are we now? Counselor Education and Supervision, 51(1), 2-16. https://doi. org/10.1002/j.1556-6978.2012.00001.x

Hatcher, L. (1994). A step-by-step approach to using the SAS system for factor analysis and structural equation modeling. Cary, NC: SAS Institute.

IBM Corporation (2016). IBM SPSS Statistics for Windows, Version 24.0 [Software de computación]. Armonk, NY: IBM.

Lilienfeld, S. O., Lynn, S. J., Namy, L. L., \& Woolf, N. J. (2011). Psicología: Una introducción. Madrid: Pearson Education S.A.

Maurino, L., Miranda, A. L., Moretti, L., FloresKanter, P. E., Casari, L., \& Medrano, L. A. (2018). Evaluación de la competencia terapéutica: Consistencia interna y acuerdo inter-jueces de la Escala de Terapia Cognitiva (CTS). Informes Psicológicos, 19(2), 95-107. http://dx.doi.org/10.18566/ infpsic.v19n2a07.

Montero, I., \& León, O. G. (2007). A guide for naming research studies in psychology. International Journal of Clinical and Health Psychology, 7(3), 847-862.

Nieves-Rosa, L. E. (2012). Homofobia al Estilo Universitario. Revista Puertorriqueña de Psicología, 23, 62-76.

Rivera-Colón, R., Ramos-Reyes, M., \& VázquezRamos, R. (2019). Las competencias del personal de consejería en rehabilitación para trabajar con poblaciones de lesbianas, gays y bisexuales (LGB). Revista Electrónica Educare, 23(3), 1-19.

Sandfort, T. G. M., de Graaf, R., \& Bijl, R. V. (2003). Same-sex sexuality and quality of life: Findings from the Netherlands Mental Health Survey and incidence study. Archives of Sexual Behavior, 32(1), 15-22. https://doi.org/10.1023/A:1021885127560

Schmitt, T. A. (2011). Current methodological considerations in exploratory and 
confirmatory factor analysis. Journal of Psychoeducational Assessment, 29(4), $\quad$ 304-321. https://doi. org/10.1177/0734282911406653

Singh, A. A., \& Dickey, L. M. (2016). Implementing the APA guidelines on psychological practice with transgender and gender nonconforming people: A call to action to the field of psychology. Psychology of Sexual Orientation and Gender Diversity, 3(2), 195-200. http:// dx.doi.org/10.1037/sgd0000179

Stangor, C. (2012). Social psychology principles. Retrieved from https://2012books.lardbucket.org/pdfs/ social-psychology-principles.pdf

Stevens, J. (2002). Applied multivariate statistics for the social sciences (4a. ed.). Mahwah, $\mathrm{NJ}$ : Lawrence Erlbaum Associates.

Tirado-Martínez, A. (2018). Prejuicio y distanciamiento social hacia las personas $L G$ en una muestra de adultos puertorriqueños padres y madres de familia. (Disertación doctoral no publicada). Universidad Carlos Albizu, San Juan, Puerto Rico.

Toro-Alfonso, J., \& Varas-Díaz, N. (2004). Los otros: prejuicio y distancia social hacia hombres gay y lesbianas en una muestra de estudiantes de nivel universitario. International Journal of Clinical and Health Psychology, 4(3), 537-551.
Vázquez-Rivera, M., Esteban, C., \& ToroAlfonso, J. (2018). Hacia una psicología libre de paños tibios: Actitudes, prejuicio y distancia social de psicoterapeutas hacia gays y lesbianas. Perspectivas en Psicología, 15(1), 15-24.

Vázquez-Rivera, M., Nazario-Serrano, J., \& Sayers-Montalvo, S. (2012). Actitudes hacia gays y lesbianas en psicoterapia de estudiantes graduados/as de psicología y psicólogos/as clínicos/as con licencia. Revista Interamericana de Psicología, 46(3), 435-446.

Wilson, F. R., Pan, W., \& Schumsky, D. A. (2012). Recalculation of the Critical Values for Lawshe's Content Validity Ratio. Measurement and Evaluation in Counseling and Development, 45(3), 197-210. https:// doi.org/10.1177/0748175612440286

Woodford, M. R., Paceley, M. S., Kulick, A., \& Hung, J. S. (2015). The LGBT social climate matters: Policies, protests, and placards and psychological well-being among LGBT emerging adults. Journal of Gay \& Lesbian Social Services, 27(1), 116-141. https://doi. org/10.1080/10538720.2015.990334

Worthington, R. L., Dillon, F. R., \& BeckerSchutte, A. M. (2005). Development, reliability, and validity ofthe Lesbian, Gay, and Bisexual Knowledge and Attitudes Scale for Heterosexuals (LGB-KASH). Journal of Counseling Psychology, 52(1), 104-118. https://doi.org/10.1037/0022-0167.52.1.104 


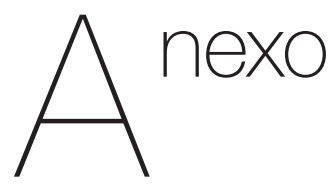

Escala de Actitudes Percibidas en Terapia hacia Lesbianas, Gays y Bisexuales

$\begin{array}{ccccc}\begin{array}{c}\text { Totalmente en } \\ \text { desacuerdo }\end{array} & \begin{array}{c}\text { En } \\ \text { desacuerdo }\end{array} & \text { Neutral } & \text { De acuerdo } & \begin{array}{c}\text { Totalmente } \\ \text { de acuerdo }\end{array} \\ \text { (1) } & \text { (2) } & (3) & \text { (4) } & \text { (5) }\end{array}$

1. Mi terapeuta se mostró incómodo cuando me preguntaba sobre mi orientación sexual.

Mi terapeuta me explicó que las actitudes negativas de la

2. sociedad por orientación sexual pueden causarme malestar psicológico.

3. Mi terapeuta exploró como las actitudes negativas de la sociedad han tenido impacto en mi vida.

4. Mi terapeuta reaccionó negativamente cuando le dije que me gustaban las personas de mí mismo sexo.

5. Mi terapeuta me cuestionó si estaba seguro de mi orientación sexual.

6. Mi terapeuta sugirió que tuviese (o continuara) relaciones heterosexuales para estar seguro de mi orientación sexual.

7. Mi terapeuta exploró sobre mis experiencias sexuales del pasado.

8. Mi terapeuta utilizaba estereotipos relacionados a mi orientación sexual.

9. Mi terapeuta exploró sobre enfermedades de transmisión sexual, como lo haría con una persona heterosexual.

10. Mi terapeuta me informó sobre su experiencia, educación y/o adiestramientos sobre orientación sexual.

11. Mi terapeuta me informó sobre recursos de apoyo apropiados para la comunidad LGB.

12. Me provocó ansiedad la forma en que mi terapeuta indagó sobre asuntos de mi sexualidad.

* Items 1, 4, 5, 6, 8 y 12 tienen corrección inversa. 